\title{
BRASIL: ÉLITES POLÍTICAS, ELECTORADO Y PERSPECTIVAS DE LA DEMOCRACIA EN LA DÉCADA VENIDERA
}

\author{
Lúcia Avelar \\ Universidad de Brasilia \\ E-mail: lucia_avelar@nol.com.br
}

\begin{abstract}
RESUMEN
En este artículo se buscan respuestas en relación a la negativa de las élites políticas brasileñas a implementar políticas de carácter socio-redistributivo, cuestionando así las razones del aumento de las desigualdades sociales en un país rico como Brasil. En el análisis de estas élites destaca la existencia de prácticas clientelistas llevadas a cabo desde el propio Estado, en las que adquiere especial relevancia la distribución regional de las élites desde el siglo XIX. Con el tiempo, la complejización de la sociedad brasileña permite un cierto cambio político que conlleva la aparición de organizaciones políticas y de asociaciones, principalmente en los Estados más ricos como son São Paulo y Río de Janeiro. Sin embargo, se mantienen los desequilibrios redistributivos entre los tres Brasiles. En un segundo momento se ponen en relación estos elementos anteriores con el grado de instrucción del electorado brasileño, en un análisis de las posibilidades de renovación de las élites y de cambio político profundo, en vistas a la aplicación efectiva de políticas redistributivas que permitan mejorar las condicioners de vida de la mayoría de la población de Brasil.
\end{abstract}

\section{I. ÉLITES Y EXPANSIÓN DEL PODER POLÍTICO: ALGUNOS PRESUPUESTOS}

Uno de los aspectos fundamentales en el debate sobre la cuestión democrática para el siglo XXI está relacionada con la capacidad de las élites políticas para realizar, de manera efectiva, políticas redistributivas en el país, presupuesto que nos lleva a discutir algunas de sus características, sus respectivos estilos 
de hacer política y el modo cómo se reproduce y realiza la expansión de su poder. La pregunta central de este trabajo es: ¿Por qué esas élites no eligieron, hasta ahora, como objetivo central de sus acciones, la implementación de políticas de carácter socio-redistributivo en nuestro país? ¿Cuáles son las razones del aumento de las desigualdades sociales en un país rico pero cuya concentración de la riqueza es una de las mayores del mundo?

El examen del tema puede ser encaminado por medio de las siguientes cuestiones: 1) ¿En qué medida presenciamos en la sociedad brasileña cambios en las bases del poder político tradicional, con la ruptura de los patrones seculares de representación de intereses y de intermediación política?; 2) ¿en qué medida es perfecta la correlación todavía existente entre el aspecto social y el aspecto político de la distribución del poder?; 3) ¿ ¿refuerzan las reglas institucionales la forma clientelista de intermediación de intereses?; 4) ¿en qué medida podemos hablar de un proceso de cambio político con la emergencia de nuevos actores y de una nueva configuración de la élite política preferencialmente vuelta hacia los aspectos sociales del desarrollo del país?

Para responder a esas cuestiones entenderemos élites políticas como los grupos que efectivamente tienen la capacidad de mando y de decisión sobre cuestiones políticas, siendo tales grupos oriundos de los canales numéricos de participación política, por medio de elecciones y partidos políticos, o de los canales corporativos del poder.

Para la discusión, nunca está de más recordar el origen de las élites brasileñas, el modo como se constituyeron y los medios institucionales que utilizaron para reproducirse y perpetuarse en el poder. Como apuntan numerosos estudios, en los orígenes de nuestra organización política, cuando la Corona portuguesa atraía a los colonizadores con la distribución de tierras, poderes e inmunidades, el resultado político fue la privatización del poder y su dispersión en localidades de enorme autonomía, dada la necesidad de protegerlas a partir del propio grupo familiar y colonizador, con vínculos de naturaleza feudal entre los propietarios y los agregados (Cintra, 1974) ${ }^{1}$. También es importante recordar que, tal como nos enseñaba Montesquieu, en los actos fundadores de una nueva forma de organización política, sus líderes moldean las instituciones políticas; en sus desdoblamientos posteriores serán las propias instituciones que moldearán a sus líderes. Por lo tanto, nuestro argumento central para responder a las cuestiones planteadas es que la interacción entre la estructura política original y la élite política resultante tuvo un papel esencial en la estructuración del poder político en Brasil y en la actual configuración de nuestras élites. El gran desafío es saber cuáles son las posibilidades de construcción democrática en un orden patrimonialista, estando el poder político fuertemente marcado por familias cuyas posiciones de centralidad social se refuerzan por las posiciones estamentales, y con prácticas de reproducción de

Los estudios sobre poder tradicional en Brasil son numerosos. Destacamos la contribución de Cintra, A. (1974). 
ese poder por medio de una red de relaciones que transmite tales posiciones y socializa políticamente a sus descendientes. En suma, en qué medida podemos esperar una actitud reformista en la dirección socio-redistributiva de un poder político tradicional y su respectiva élite política tradicional ${ }^{2}$, a no ser por la búsqueda de nuevos fundamentos de legitimidad de este poder frente a las presiones por el cambio ejercidas por los nuevos actores sociales.

Nuestra hipótesis es que si el país cambió su cara económica, la estructura política todavía presenta hoy fuertes trazos de su estructura fundadora, o sea, sus principales representantes; los miembros de nuestras élites, o descienden del poder político tradicional, o son miembros de familias oligárquicas emergentes, utilizando todos ellos la forma clientelista de intermediación de intereses que les garantiza la continuidad, particularmente en las regiones de menos desarrollo. Estas últimas, en virtud de circunstancias históricas peculiares, utilizaron los mismos canales institucionales de las élites tradicionales para ascender al poder. Es del Estado de donde retiran los recursos financieros para fortalecerse en el ámbito local; cuanto mayor es su posición en el centro, mayor es su poder en la periferia.

\section{Élites, posición social y posición política}

El caso brasileño no es muy diferente al de otros países cuyas élites se estructuraron a partir del dominio de la gran propiedad de la tierra ${ }^{3}$. Las oligarquías brasileñas fundan su poder en la clase agraria. Organizadas en bases regionales, dominaron la política nacional desde su inicios, pasando por la Independencia, la República, la era Vargas, sobreviviendo a la era populista y al régimen autoritario instalado en 1964 . Hasta la República Velha dominaron el Estado como élites agrarias; con la centralización fiscal del régimen Vargas (1930) y con el régimen de 1964, transfieren su actuación para el centro del Estado, retirando de ahí recursos para mantener a sus electores en el nivel

${ }^{2}$ Las élites políticas tradicionales perpetúan la "política tradicional», que es un patrón de política en el que el poder está altamente concentrado, el acceso a las arenas de decisión es restringido, los niveles de competición estrictamente regulados, teniendo como fundamento el regionalismo, el personalismo y el clientelismo. El concepto identifica una clase que no ocupe una posición prominente en la estructura productiva y cuyo poder no deriva únicamente de los recursos económicos. El poder es transmitido por linaje familiar, y tiene como base la propiedad de la tierra o el acceso a los recursos del Estado (Hagopian, 1996).

3 Oliveira Vianna evidencia la semejanza de la fase colonial de nuestra historia con lo que ocurrió en Europa durante los primeros siglos del régimen feudal. Tal como ocurría entre los señores medievales, en razón de la ocupación de sus dominios (haciendas y áreas cultivadas) hubo una especie de guerrillas interfeudales. Con el paso del tiempo, esos señores, que también se convirtieron en personalidades políticas, expresaban su identidad a través de los conflictos inter-élites, presentes, todavía hoy, en varias partes del país, siendo una de las razones por las cuales las numerosas siglas partidarias brasileñas no son sino simples nombres para la transmisión de intereses de los grupos locales. 
local. Es de ese modo que las élites agrarias pasaron a ser élites estatales ${ }^{4}$. La ruptura de este modelo podría haber ocurrido en el proceso de cambio económico, con el paso de la economía de base agraria a la capitalista y con la progresiva expansión del sufragio, pues la correspondencia entre posición social y posición política es una fórmula que todavía persiste en la mayoría de la sociedades "cerradas", en las cuales la presencia en los gobiernos y cargos de poder es un privilegio de la posición disfrutada en la sociedad civil y la participación en la política una especie de derecho automático de los ocupantes de determinado status social (Pizzorno, 1996). Pero, en el caso brasileño, los cambios son lentos debido a la vitalidad de las élites tradicionales.

La industrialización, el desarrollismo, el capital internacional, no consiguieron diluir el poder de esos grupos en el aparato estatal, en parte porque el desarrollo económico bajo la forma de un capitalismo de Estado hizo de la economía pública el foco de las actividades públicas, y las funciones de intermediación política de naturaleza clientelista se profundizaron, particularmente en las regiones en que la economía pública superaba la influencia de las élites económicas privadas. La práctica de esta forma de intermediación política es conocida por el nombre de "Clientelismo de Estado", una formación histórica y estructural constituida por operaciones desequilibradas de cambio de recursos materiales obtenidos en el ámbito del Estado y usados para la obtención de apoyo político. La subordinación de la población es su rasgo más característico y puede tomar varias formas, desde la compra de votos hasta movilizaciones 5 controladas como en los regímenes autoritarios (Fox, 1994).

El estudio del clientelismo, de mala reputación entre los intelectuales brasileños, ganó fuerza en los últimos años en el ámbito académico. Se llegó a la conclusión de que el tema ha sido tratado con estereotipos y poca investigación empírica (Bezerra, 1999; Leff, 1977). Recuperando a grandes teóricos de las décadas anteriores (Roniger y Eisenstadt, 1980; Eisenstadt y Lemarchand, 1981; Kaufman, 1974; Schmidt, 1977), las investigaciones en la década de 1990 llaman la atención sobre la pertinencia de esta temática cuando se trata de analizar lo que se denomina "política tradicional» y «élites políticas tradicionales», en los países que tuvieron el poder político estructurado a partir de la gran propiedad de la tierra, como es el caso de Brasil, y cuyas élites continuaron sobreviviendo incluso cuando la tierra como recurso de poder fue perdida, e incluso después de los procesos de industrialización y urbanización (Hagopian, 1996). Con la concentración de poder en el Estado, en el proceso de "capitalismo de Estado», esas élites supieron cómo manipular el sufragio a su favor y alojarse en el aparato del Estado, retirando de allí sus recursos para las

${ }^{4}$ La bibliografía sobre el tema es amplia. En numerosos estudios encontramos evidencias de cómo el cambio institucional del Estado promovido por Vargas, en un intento de corregir el regionalismo de la Velha República, no tuvo como resultado sino el alojamiento de los líderes locales y regionales en el centro del aparato estatal.

${ }^{5}$ Clientelismo y protección son recursos utilizados por las oligarquías dominantes para el control electoral, impidiendo que la población se organice en movimientos autónomos. 
operaciones de protección (patronagem) en el ámbito local. De un clientelismo local se pasó a practicar un clientelismo de Estado. La política clientelista y las formas modernas de intermediación de intereses no existen en formas puras, en zonas demarcadas territorialmente, pero coexisten en las diversas regiones y en el Estado brasileño.

El clientelismo practicado en las primeras décadas del siglo XX tenía como recurso la propiedad de la tierra. El clientelismo de posguerra combinó elementos de la política tradicional y de la moderna. Tradicional, porque continuó siendo practicado por los descendientes de las familias cuyo poder venía del tiempo del Imperio, perpetuándose en la República, resistiendo a la centralización varguista de 1930, revitalizándose en el período democrático-representativo de 1946 a 1964, apartados temporalmente en los primeros años del régimen militar (1964-1985) pero recuperando su fuerza en la transición política (1984/1986) y en la era pos-autoritaria. Los electores, por su lado, continuaban demostrando más lealtad a los individuos descendientes de esas familias que a los partidos. Moderno, porque, incluso con mayor integración territorial y mayor competición interpartidaria, los políticos locales fueron capaces de sellar alianzas con autoridades federales, en un sistema integrado verticalmente, definido por la competición de recursos clientelistas, teniendo como centro los gabinetes, secretarías y subsecretarías de la burocracia del Estado, en una lógica de acción que era la de liberar recursos del centro a cambio de apoyo político local. El network ${ }^{6}$ clientelista vincula el jefe local al centro político nacional, y el clientelismo de Estado es la nueva forma que sustituiría al clientelismo tradicional. El clientelismo penetró los partidos de masas definiendo la naturaleza de esos partidos, en la misma línea de utilizar los recursos públicos para las operaciones clientelistas (Hagopian, op. cit., p. 61). Además de las dimensiones citadas del clientelismo tradicional y de Estado, otros estudios utilizan otra dimensión, la del semi-clientelismo, en un esfuerzo de teorización y análisis empírico de realidades de los países en los cuales sobrevive la "política tradicional». Es importante distinguir clientelismo de corrupción. Aunque los focos de corrupción en las distintas instancias del Estado sean evidentes, clientelismo es el intercambio de favores por apoyo político; corrupción es la maximización del interés monetario, siendo el objetivo de las operaciones el dinero público apropiado para fines personales (Della Porta, 1994).

La práctica del clientelismo, originaria de la estructura patrimonialista del Estado portugués, se legitimó en las décadas recientes por la vía institucional en el sistema de representación proporcional, cuyas reglas fueron idealizadas en los años de Vargas e incorporadas a la Constitución de 1946. Algunos de sus aspectos fueron profundizados por uno de los presidentes militares, en 1976, con el Paquete de Abril. Ese conjunto de reglas de representación es el propio coronamiento de la victoria de las oligarquías de base regional y local del poder. En palabras de Martins (1994): «El Estado brasileño aún tiene por base

${ }_{6}^{6}$ Todas las palabras que aparecen en inglés constan así en el original en portugués. (N. del T.) 
relaciones políticas extremadamente atrasadas, como las del clientelismo y las de la dominación tradicional de base patrimonial, del oligarquismo. En Brasil, el atraso es un instrumento de poder».

Son varios los estudios sobre la persistencia del poder oligárquico en Brasil. Citamos, entre otras, las investigaciones que muestran la red familiar ocupando los lugares de poder tanto en los cargos electos como en los de las burocracias, secretarías y subsecretarías estaduales y federal ${ }^{7}$. Las redes clientelistas y de protección (patronagem) siguen siendo las actividades utilizadas por las oligarquías dominantes para el control electoral y también para impedir la organización de movimientos autónomos, una dinámica que, en un gran número de municipios, presenta pocos elementos de imprevisibilidad, ya que la relación fundamental es la de fidelidad a cambio de recursos materiales. Ejemplos de cambio son los de las capitales de estados y regiones de mayor desarrollo, con variadas formas de movimientos urbanos y organizaciones populares, profesionales, reivindicando sus intereses y convirtiéndose en interlocutores tanto de la sociedad como del Estado. Más aún, el difícil paso del clientelismo a la ciudadanía es uno de los grandes desafíos políticos de la actualidad (Fox, op. cit.).

\section{CAMBIO POLÍTICO}

En sociedades como la brasileña, con una fuerte diferenciación entre las clases —un enorme distanciamiento entre los segmentos de la clase media y alta respecto a los segmentos populares_-, la cuestión crucial es saber en qué condiciones podrá darse la ruptura del monopolio del poder político a partir del status adscrito y la emergencia de «nuevas élites». Entre otros factores, apuntamos los siguientes:

\section{La organización politica}

Nuevos canales de acceso al poder se viabilizaron por medio de la organización política, con el ascenso de representantes de otros segmentos que, incluso sin tener posiciones de centralidad social, consiguieron reivindicar el derecho de los ciudadanos comunes en la promoción a la esfera política. Esto no sería posible si los grupos que están en el poder no tratasen de fortalecerse por medio de alianzas con nuevas fuerzas populares que, en última instancia, los fortalecen con nuevos fundamentos de legitimidad (Pizzorno, 1972). Los datos sobre tal fuente de cambio serán presentados más adelante.

Nótese el caso de Minas Gerais, cuyas familias políticas son originarias de regiones fácilmente identificables, configurando una cuasi distritalización de la representación. Los estudios son varios pero, entre otros, citaríamos a Dulci (1992), Canedo (1993) y Carvalho (1969). 


\section{El sufragio}

La ampliación progresiva del sufragio introdujo el principio de una lógica igualitaria de modo que los individuos pudieran practicar los mismos derechos ante al Estado. Si bien la institución del voto se convirtió en un canal potencial de acceso al poder para luchar contra la estructura de desigualdades de status y poder, el proceso de cambio es lento: algunas sociedades cambiaron sus respectivas élites por medio del voto; otras vieron a sus élites perpetuarse en el poder por la manipulación del sufragio a su favor, utilizando para esto los recursos del Estado. Aún hoy es enorme el predominio de los partidos políticos tradicionales y de configuración oligárquica en el ámbito municipal, como nos muestran los números de más abajo, un cuadro evidente de fuerza del poder tradicional incluso en contextos de relativo desarrollo y de modernización económica.

Número de Ayuntamientos obtenidos por los partidos politicos, clasificados según su tendencia ideológica en las regiones clasificadas según el Índice de Desarrollo Humano (IDH), $1996^{\circ}$

\begin{tabular}{|c|c|c|c|}
\hline Partidos politicos & $\begin{array}{c}\text { IDH por encima } \\
\text { de } 0,8 \\
(\%)\end{array}$ & $\begin{array}{c}\text { IDH entre } \\
0,7 \text { y } 0,8 \\
(\%)\end{array}$ & $\begin{array}{c}\text { IDH por debajo } \\
\text { de } 0,7 \\
\text { (\%) }\end{array}$ \\
\hline 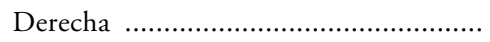 & 38 & 46 & 54,5 \\
\hline 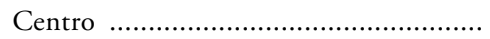 & 44 & 45 & 33 \\
\hline 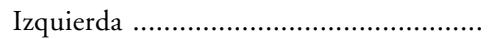 & 17 & 8 & 12 \\
\hline Otros & 1 & 1 & 1,5 \\
\hline
\end{tabular}

FUENTE: Tribunal Superior Electoral e Informe IPEA/PNUD, 1998. Investigación de Avelar y Lima, 2000.

La representación política en la Cámara Federal sigue el mismo patrón: en las regiones de más alto IDH hay un relativo equilibrio en la representación partidaria de las diferentes tendencias: la derecha tiene un 38 por 100; el centro, un 31 por 100, y la izquierda, un 31 por 100 . En las regiones de IDH

${ }^{8}$ Los partidos fueron clasificados del siguiente modo: derecha: el Partido del Frente Liberal (PFL), el Partido Liberal (PL), el Partido Trabajador Brasileño (PTB), el Partido Progresista Brasileño (PPB), el Partido Social Demócrata (PSD), el Partido Social Cristiano (PSC), y otros partidos menores que en las elecciones municipales de 1996 obtuvieron el 2,6 por 100 de los votos. Los partidos de centro son el Partido del Movimiento Democrático Brasileño (PMDB), el Partido de la Social-Democracia Brasileña (PSDB). Los partidos de izquierda: el Partido de los Trabajadores (PT), el Partido Democrático Trabajador (PDT) y el Partido Socialista Brasileño (PSB).

9 Instituto de Investigaciones Económicas Aplicadas (N. del T.). 
entre 0,7 y 0,8 , la derecha tiene un 52 por 100 ; el centro, un 35 por 100 , y la izquierda, un 13 por 100; en las regiones cuyo IDH es el más bajo, menor de 0,7 , la derecha tiene un 48 por 100; el centro, un 35 por 100, y la izquierda, un 17 por 100. En estas cifras hay otra dimensión que debe ser destacada: la sobre-representación de los estados menos poblados en la Cámara Federal, garantizada por la Legislación Proporcional. Además del aspecto de la subrepresentación de los ciudadanos de todo el país (Stepan, 2000) ${ }^{10}$, está el aspecto de la hegemonía política. Si son las fuerzas políticas tradicionales que dominan las regiones de menos desarrollo y si son ellas las que están sobrerepresentadas en el centro, no existen constraints suficientes que garanticen un libre juego de intereses con las respectivas poblaciones representadas. Lo que se produce es una restricción de intereses que garantiza la continuidad de la política tradicional en esas regiones. Como demuestran las encuestas de opinión, cuyos datos apuntan una enorme insatisfacción y desconfianza de la población hacia los representantes políticos, esos datos reflejan con claridad que, para amplias clases de la población, el clientelismo de Estado no da cuenta de las demandas de una sociedad ahora urbana, compleja y profundamente desigual (Linz y Stepan, 1999). Para Linz, el resultado es una "república federativa de insatisfechos», por la evidencia de que las estructuras de oportunidades no son iguales para todos (Linz, 1996). Serían necesarias otras formas de liderazgo para que las políticas redistributivas fueran implementadas de manera favorable a los segmentos hasta entonces descuidados por el Estado y sus élites. Es ésta la percepción fundamental que ha llevado a la ampliación de las bases de poder de los partidos originarios de la sociedad organizada.

\section{La crisis urbana}

La crisis originada por el surgimiento de las masa urbanas puede ser considerada como un hecho histórico que provocó procesos de organización política y de participación, como la emergencia de nuevos actores presionando para la entrada en el cerrado círculo del poder. Fue con la crisis urbana que vimos la constitución de nuevas fuerzas políticas expresadas en organizaciones de carácter asociativo, con nuevas formas de representación de intereses. Sin ningún atisbo de duda, los cambios en el cuadro partidario brasileño, que por primera vez en la historia representa un conflicto real inter-élites, es una de las señales más evidentes de ese cambio, tal y como señalaremos más adelante.

${ }^{10}$ Alfred STEPAN, Towards a new comparative analysis of democracy and federalism, Mimeo, Oxford. 


\section{Los desequilibrios redistributivos}

El desarrollo y la industrialización ocurridos en el país se dieron con un fuerte desequilibrio redistributivo, tanto a nivel inter-clasista como en el interregional, dificultando todavía más el proceso de construcción institucional del Estado y la expansión de la solidaridad de ámbito territorial. Para numerosos segmentos de la sociedad quedaría claro que el desarrollo se producirá para algunos, «los de dentro», en oposición a «los de fuera» en relación a la esfera de intervención del Estado. Esta cuestión se hizo aún más evidente con la redemocratización del país, pues, aunque formalmente restablecida la democracia política, la democracia social estaba lejos de ser alcanzada. La cuestión democrática pasó a tener en la cuestión social uno de sus mayores obstáculos (Reis, 1994; Keck, 1991; Avelar, 2000).

Para ilustrar esto tomemos datos sobre la curva de distribución de la renta en Brasil. Según lo que nos informa el Informe sobre Desarrollo Humano de 1998, entre 1960 y 1990 la renta de los 20 por 100 más ricos aumentó un 11 por 100 , mientras la renta del 50 por 100 de personas más pobres disminuyó un 6 por 100. La pobreza tiene un componente claramente regional, siendo más elevada en las regiones Norte (43 por 100) y Nordeste (46 por 100), reduciéndose en dirección al Sur (20 por 100). Los porcentajes son también elevados en la población rural (39 por 100) y en las grandes ciudades, tal como ya apuntamos, y así nos muestran los datos relacionados con la «metropolización de la pobreza", ya que los pobres de las metrópolis aumentaron expresivamente. La polarización y la pobreza extrema se encuentran en el Nordeste rural y en las metrópolis del Sudeste. El cuadro resultante se aproxima a un apartheid social.

La población brasileña está compuesta por un 55 por 100 de blancos, un 4,9 por 100 de negros, un 39 por 100 de mulatos y un 0,5 por 100 de asiáticos. Los mulatos y negros sumados representan el 43,9 por 100 de la población. La renta media de los hombres negros y mulatos es del 63 por 100 y 68 por 100 , respectivamente, de los hombres blancos. La renta de las mujeres negras y mulatas corresponde a 68 por 100 de la renta de las mujeres blancas. El factor racial se combina con la dimensión regional: en el Sudeste y en el Sur del país predominan las personas de color blanco (83 y 66 por 100, respectivamente); en las regiones del Norte y del Nordeste, los mulatos (71 y 65 por 100 , respectivamente). En otras palabras, los negros y mulatos están concentrados en las regiones que presentan el menor nivel de renta per capita. Los análisis han mostrado que las diferencias de renta y acceso a la educación son un resultado simultáneo de por lo menos dos factores, la raza y la región. Estos factores combinados son los mayores responsables por el distanciamiento entre las clases sociales. Sin hablar de la dimensión sexo: las estadísticas muestran que las mujeres trabajadoras ganan salarios que son en media 63 por 100 menores que los de los hombres para los mismos trabajos. La gravedad del hecho es enorme porque el número de familias encabezadas por mujeres aumentó un 13 por 100 entre 1980 y 1991 (PNUD/IPEA, 1998). 
En el ámbito de las políticas públicas, uno de los grandes desafíos es el de la alfabetización de adultos y el rescate de una deuda educacional que alcanza a miles de brasileños y hiere los fundamentos y los derechos de ciudadanía en cualquier país. Las clases no educadas han sido la base de la política populista y clientelista que afecta directamente a la eficiencia de los gastos públicos en el ámbito social. Un cuadro de estas diferencias será presentado más adelante.

\section{ALGUNOS PRESUPUESTOS SOBRE LA AMPLIACIÓN DE LA PARTICIPACIÓN Y LA EMERGENCIA DE NUEVOS ACTORES POLÍTICOS}

Las transformaciones de la política son, en gran medida, el resultado de una serie de conquistas, existiendo un lag incontestable entre los cambios económicos y los cambios políticos. Una de las conquistas de la política brasileña reciente es la ampliación de la participación. A la Iglesia católica puede ser atribuido el mérito de las primeras organizaciones de las comunidades de base, desde los años cuarenta, construyendo verdaderos cinturones de asociaciones, que dieron el origen de los movimientos sociales urbanos ${ }^{11}$. En los años autoritarios, particularmente en la década de 1970, proliferaron los movimientos sociales: si hay una referencia histórica para el empowerment de la sociedad, ésta puede ser apuntada para esos años ${ }^{12}$.

Para que se dé el empowerment de los grupos que se encuentran en condiciones de desigualdad estructural en la sociedad civil y política, la primera condición es la formación de las «áreas de la igualdad», una precondición para que los valores de los individuos que comparten el mismo sistema de intereses se aglutinen en sistemas de solidaridad. Sus participantes se conciencian de que, potencialmente, hay en la sociedad una regla igual para todos, pero que sólo por medio de acciones colectivas y la presencia en la élite política habrá una actuación efectiva para que las desigualdades estructurales sean negadas y se definan nuevas estructuras de intereses.

Se trata de un proceso lento: además de identificar las respectivas «áreas de igualdad», el individuo deberá vencer sus dilemas íntimos relacionados con el compromiso en las actividades políticas. Es fundamental la acción pedagógica de los sindicatos, movimientos y partidos que convencen a potenciales partici-

11 Todavía son escasas las investigaciones documentales en los obispados, particularmente en los más antiguos. En ellas, por lo que sabemos, encontramos importantes documentos que registran la acción de la Iglesia haciendo aclaraciones a las poblaciones sobre los derechos de ciudadanía. Uno de esos obispados a los que nos referimos es el del municipio de Natal, en Rio Grande do Norte, que invocaba en la década de los cuarenta una mayor participación política de la población pobre rural y urbana.

${ }_{12} \mathrm{El}$ asociacionismo bajo el régimen autoritario se encuentra documentado en numerosas tesis y disertaciones de maestría y doctorado de las universidades brasileñas, particularmente aquellas defendidas en las universidades católicas del país. 
pantes sobre su posición de clase real en la sociedad ${ }^{13}$. Por tanto, la «cultura de participación» se abre paso en una sociedad de poder concentrado como una conquista progresiva, con fuertes elementos de socialización política y gracias a la utilización de recursos de toda naturaleza: desde las campañas de concienciación tanto de la posición social compartida por distintos individuos como de la condición de desigualdad estructural en el ámbito de las clases y del Estado, a la implicación activa en ritos electorales y movimientos políticos. La participación sólo pasa a ser un valor después de una larga vivencia entre pares, con la identificación de la condición de desigualdad estructural en el ámbito de las clases y del Estado.

El ascenso de la "política de la sociedad organizada" — los movimientos sindicales, los movimientos sociales vinculados a la Iglesia católica, las asociaciones de barrio, las asociaciones de amas de casa, los movimientos de los Sin-tierra, los movimientos ecologistas, los movimientos de mujeres, los movimientos negros, los fórums para alfabetización y educación, los movimientos de defensa de los favelados y la proliferación de las Organizaciones No Gubernamentales (ONGs) - tuvo, naturalmente, desdoblamientos en el área política formal, a través de los canales partidarios. Nuevas identidades colectivas fueron moldeadas, y hoy es indiscutible la presencia de la acción colectiva guiada por líderes profesionalizados. Sin sombra de duda, la sociedad ahora está mucho más abierta que en cualquier otro período de nuestra historia con, por lo menos, dos lógicas en competición: la del Brasil desorganizado y la del Brasil organizado, a la búsqueda de relaciones más institucionalizadas en la política.

\section{Asociacionismo, redes civicas, organización colectiva}

La "política de la sociedad organizada» tiene por principio la formación de redes cívicas en la sociedad con representación en la política, con el objetivo de negociar en la arena política formal los intereses colectivos. Sus miembros y respectivos representantes tienen, en la cuestión pública, el apoyo de su acción. Tal estilo de hacer política corresponde a un proceso de transformación en el nivel más estructural y profundo, con la ascensión de nuevas fuerzas políticas.

La constitución de redes cívicas es crucial para la emergencia de nuevas élites. Es de ese modo que la sociedad brasileña viene abriéndose hacia una probable superación de las élites políticas cuya orientación es la de la política tra-

${ }_{13}$ Raramente hay una correspondencia entre la posición objetiva de clase a la que pertenece el individuo y su auto-identificación de clase. En general, la persona tiende a hacer una evaluación poco realista de su clase, lo que dificulta su discernimiento en cuanto a los líderes que realmente representarán sus intereses. Es con la participación política que se puede alcanzar la percepción de su condición social real y de los liderazgos que la representan. La otra vía es la de una escolaridad mayor. 
dicional $^{14}$. La literatura brasileña sobre el tema es amplia, caracterizada por una verdadera maraña conceptual, la mayoría de las veces de conformación «doctrinaria salvacionista», y enfatizando excesivamente el peso político de los respectivos grupos de tipo organizacional de gran complejidad (Azevedo y Prates, 1996; Mainwaring y Viola, 1987). Pero lo que es importante resaltar es que fue progresiva la emergencia de movimientos reivindicativos de todo tipo. Al lado de la proliferación de los movimientos sociales urbanos, se expandieron las organizaciones de la sociedad civil: las organizaciones sin fines lucrativos, las de caridad y asistencia, las asociaciones voluntarias, las organizaciones no gubernamentales, las fundaciones privadas, al lado de iglesias de variados credos que acabarían teniendo funciones articuladoras en la sociedad. Vimos, también, la emergencia de la planificación participativa en el ámbito de la política urbana y, allí donde existían "asociaciones comunitarias», se inicia una interacción con los programas públicos; donde no existían, se inducía su creación. La práctica de la planificación participativa en algunos ayuntamientos del país, en general de las regiones Sur y Sudeste, han tenido un efecto real de mayor participación de la sociedad en la decisión del presupuesto público y, al mismo tiempo, socializando políticamente a la población sobre los derechos de ciudadanía.

Tomemos algunas informaciones para las ciudades de São Paulo y Río de Janeiro. Entre 1920 y 1986 fueron creadas en torno a 8.400 asociaciones (deportivas, culturales, de beneficiencia, etc.), siendo un 68 por 100 de ellas constituidas después de 1970. Si en el inicio el énfasis organizacional era en el campo de los deportes, en las últimas décadas lo que se dio fue la apertura organizacional para otras modalidades asociativas. E incluso, la década de 1970 fue decisiva en el reordenamiento social y organizacional: tal como se ve en la tabla de abajo, de las quince categorías organizacionales de movilización reciente, diez están ocupacionalmente definidas.

Así como puede desprenderse de los datos relativos a São Paulo, también los de Río de Janeiro apuntan las mismas tendencias: de las cerca de 5.800 asociaciones creadas entre 1946 y 1987, un 67 por 100 surgieron después de 1970. Es importante observar que del total de las asociaciones de trabajadores manuales creadas desde 1946, el 63 por 100 fueron creadas entre 1971 y 1987. Creció también el número de asociaciones ocupacionales, en contraposición a aquellas de interés general, lo que evidencia una nueva dinámica organizacional, sea del lado de los propietarios, sea del lado de los trabajadores, funcionarios, etc.

${ }^{14}$ La afirmación toma como referencia los datos de una investigación que vengo realizando, titulada Desarrollo y Poder Político en Brasil. Los primeros resultados señalan áreas geográficas en las cuales la competición política es, hegemónicamente, de naturaleza oligárquica, y la competición electoral se caracteriza por el conflicto inter-élites. En otras regiones, sin embargo, son claros los datos sobre la introducción del pluralismo político, dándose la competición realmente entre élites de origen distinta. 
Asociaciones creadas en São Paulo y Río de Janeiro en las décadas de 1970 y 1980

\begin{tabular}{|c|c|c|c|}
\hline \multicolumn{2}{|c|}{$\begin{array}{c}\text { Porcentaje de asociaciones creadas en São Paulo } \\
\text { entre } 1970 \text { y } 1986 \text { en relación al total } \\
\text { de las creadas desde } 1920\end{array}$} & \multicolumn{2}{|l|}{$\begin{array}{l}\text { Porcentaje de asociaciones creadas en Río } \\
\text { de Janeiro entre } 1971 \text { y } 1987 \text { en relación } \\
\text { al total de las creadas desde } 1946\end{array}$} \\
\hline Comunitarias ................. & 98 & Comunitarias & 91 \\
\hline Profesionales de la salud ...................... & 93 & Vecinos ............................... & 85 \\
\hline Vecinos & 91 & Profesionales de la salud ....................... & 83 \\
\hline Abogados ... & 88 & 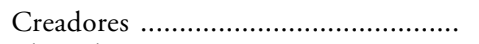 & 81 \\
\hline Deficientes & 83 & Abogados & 76 \\
\hline 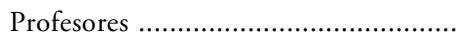 & 82 & 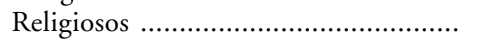 & 75 \\
\hline Padres, madres y alumnos .................. & 81 & 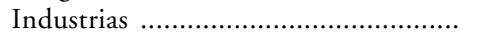 & 71 \\
\hline 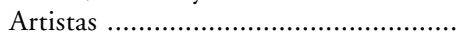 & 79 & 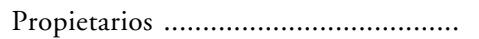 & 70 \\
\hline 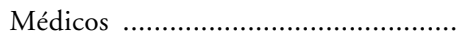 & 77 & 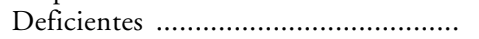 & 68 \\
\hline 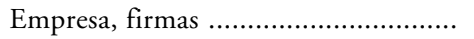 & 74 & Trabajadores no manuales ................... & 67 \\
\hline 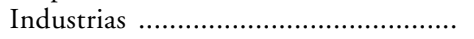 & 72 & 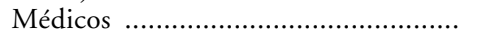 & 67 \\
\hline 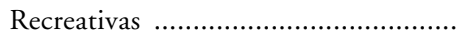 & 70 & 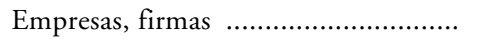 & 66 \\
\hline Trabajadores no manuales .................. & 70 & 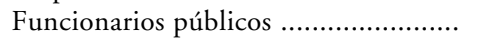 & 66 \\
\hline Trabajadores manuales ....................... & 70 & & \\
\hline 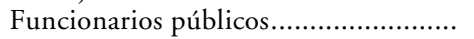 & 70 & & \\
\hline
\end{tabular}

Fuente: Archivo del Registro Civil/Publicación PNUD-IPEA, 1997.

Otro dato de importancia innegable se refiere al número de sindicatos creados entre 1900 y 1988: 9.100. De ese total, un 64 por 100 se constituyeron entre 1960 y 1988 . De ese mismo total, un 51 por 100 son urbanos: el 31 por 100 de ellos son de empleadores y el 53 por 100 de empleados. Los datos respecto a los sindicatos rurales son todavía más sorprendentes: de los 1.800 sindicatos de empleadores rurales, el 71 por 100 emergieron entre 1960 y 1988, en cuanto a los 2.700 sindicatos de empleados rurales creados entre 1900 y 1988, el 96 por 100 surgieron entre 1960 y 1988.

\section{¿Quién participa?}

Muchos de los que participan vienen de los segmentos de mayor escolaridad, tal como muestra la encuesta reciente del IBGE (Instituto Brasileño de Geografía y Estadística).

La escolaridad es un factor que afecta al grado de participación: ésta crece con el aumento del número de años de estudio, hasta el punto de que la proporción es tres veces mayor entre las personas de once años de estudio o más. Las formas de participación también son distintas según el nivel de instrucción: en huelgas, por ejemplo, la tendencia creciente se da con el aumento de la escolaridad. En las reuniones de grupos locales —asociaciones de vecinos, por ejemplo-, la participación es inversamente proporcional al nivel de escolaridad. 
Regiones metropolitanas de Recife, Salvador, Rio de Janeiro, Belo Horizonte, São Paulo y Porto Alegre: personas de dieciocho años o más, según los años de estudio

\begin{tabular}{|c|c|c|c|}
\hline Años de estudio & Asociadas & Porcentaje & No asociadas \\
\hline Sin instrucción y menos de 4 años ........ & 23.462 & 4 & 4.111 .279 \\
\hline 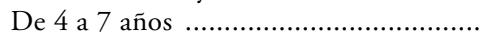 & 72.746 & 13 & 7.429 .124 \\
\hline 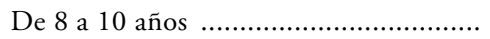 & 56.069 & 10 & 3.863 .793 \\
\hline 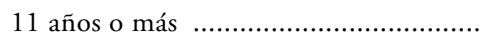 & 400.635 & 73 & 6.518 .410 \\
\hline TOTAL & 552.912 & 100 & 21.922 .606 \\
\hline
\end{tabular}

FUENTE: IBGE: Encuesta mensual de empleo, abril de 1996.

Un dato importante a ser destacado es el del asociacionismo en las regiones metropolitanas cuando se comparan entre sí: Porto Alegre (extremo sur), la región metropolitana cuya población presenta los mejores índices de escolaridad del país, obtiene tasas de participación mayores que las demás en todas las formas de asociacionismo, con la tasa de participación en sindicatos superando, incluso, la del área metropolitana de São Paulo. Además de esto, aquella región metropolitana congrega la misma proporción de personas en el asociacionismo de tipo comunitario y sindical. Una de las conclusiones posibles frente a los datos es que la formación de redes cívicas, con la emergencia de nuevos líderes políticos y nuevas formas de intermediación política se debe, entre otros factores, a la mejora de las condiciones sociales de la población pero, principalmente, en lo que se refiere a la educación. Cuanto mayor es la proporción de personas que consiguen ascender a niveles más altos de escolaridad, llegando a alcanzar por lo menos once años de estudio, mayor es su tendencia a participar en la política.

\section{Corporativismo}

Después de siglos de una sociedad controlada por el Estado y por las élites locales, hoy podemos hablar de algún grado de corporativismo social, y no sólo de corporativismo estatal. Este término debe ser entendido en su perspectiva positiva, es decir, como indicador de la capacidad de la sociedad para articularse en organizaciones que defiendan colectivamente sus intereses. Reis (1989), en una formulación altamente provocativa, dice: "Al contrario de lo que pretenden las corrientes tendentes a denunciar el corporativismo brasileño, en Brasil falta corporativismo». El autor nos llama la atención sobre el hecho de que, con mayor asociacionismo, se producen mayores oportunidades de cambios en las relaciones entre élites manipuladoras y masas amorfas, principalmente por el nuevo cometido social y político que las redes cívicas vienen presentando en su dinámica política. 


\section{LÍMITES AL CAMBIO POLÍTICO}

El desarrollo desigual del país, al promover graves distorsiones en la distribución de la riqueza, generó, como todos sabemos, un país profundamente diversificado, cuyo carácter puede ser expresado en la imagen de los tres Brasiles (PNUD/IPEA, 1996). El mapa retrata la imagen de estos tres sub-países, cuyos patrones diversos de desarrollo configuran una fuerte diferenciación regional, con consecuencias en las distintas estructuras del poder político. Para la confección del mapa se utilizó el Índice de Desarrollo Humano, siguiendo la clasificación de los estados en cada sub-región conforme a los indicadores de salud, educación y renta.

\section{ESTADOS y Territorios}

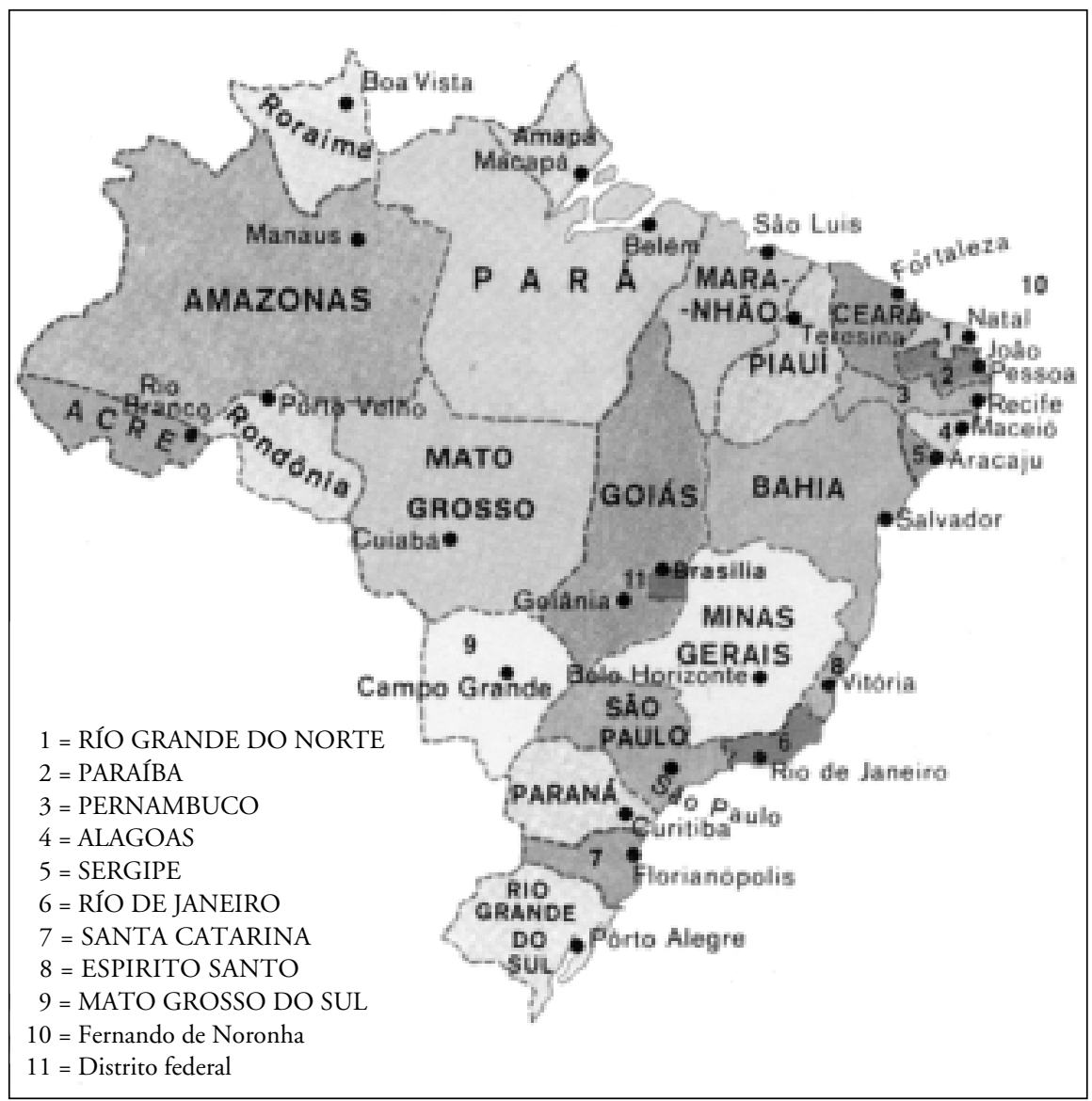


Los siete estados del sur del país (Rio Grande do Sul, Santa Catarina, Paraná, São Paulo, Río de Janeiro, Espírito Santo, Mato Grosso do Sul) más el Distrito Federal, presentan un nivel de desarrollo humano cuyo índice es superior a 0,8; forman el llamado primer Brasil. La franja que se extiende en dirección Noroeste, a partir del estado de Minas Gerais —incluyendo los estados de Goiás, Mato Grosso, Rondônia, Amazonas y Roraima-, más el de Amapá, con índices de desarrollo humano situados entre 0,7 y 0,8 , constituyen el segundo Brasil. La otra franja, que reúne el estado de Pará, de la región Norte, y los estados del Nordeste - Pernambuco, Ceará, Paraíba, Rio Grande do Norte, Sergipe, Bahía, Maranhão y Piauí-, más el de Acre, cuyos índices respectivos son inferiores a 0,7, constituyen el tercer Brasil.

Son numerosas las cuestiones de política relacionadas con las diferencias sociales en cada una de las tres subregiones ${ }^{15}$. Datos como el número medio de años de estudio del electorado en cada sub-región, nos ayudan a reflexionar sobre tales diferencias. Los electores del primer Brasil tienen, de media, 4,23 años de estudio; los del segundo Brasil, 3,02, y los del tercer Brasil, 2,18 años de estudio (Informe PNUD/IPEA, 1996) ${ }^{16}$.

Veamos otros datos relacionados con el perfil educacional del electorado brasileño y la concentración del electorado de menor grado de instrucción, según las tres sub-regiones del país.

La encuesta PNAD/IBGE de 1996 nos informa sobre el perfil educacional de la población brasileñas de veinte años y más. Los datos que teníamos anteriormente son de 1990, obtenidos en el Tribunal Superior Electoral, y optamos por tomar aquellos ofrecidos por el IBGE, más actuales, incluso si ignoran el electorado entre dieciséis y diecinueve años. Una justificación para tal opción se debe al hecho de que, comparándose éstos con los datos del TSE de 1990, se concluye que el perfil educacional del elector, en términos relativos, no sufrió modificaciones muy sustanciales.

Entre los individuos de veinte años y más, el 16 por 100, o aproximadamente 14.000.000, no tuvieron instrucción o cuentan con menos de un año de instrucción. Con períodos de uno a tres años de instrucción existe un 18,4 por 100 (16.618.725 personas); de cuatro a siete años, son un 31,5 por 100 (28.503.374 personas). El total acumulado con menos de 7 años de instrucción es de 59.379.126 individuos en todo el país, o el 66 por 100 del total de este grupo de edad.

Del total de individuos de veinte años y más sin instrucción o con menos de un año, el 47 por 100 de ellos se encuentran en el tercer Brasil; el 37 por 100, en el primer Brasil, y el 17 por 100, en el segundo Brasil.

15 En el interior del país, particularmente en los estados localizados en el segundo y tercer Brasil, hay municipios muy extensos con poblaciones escasas.

${ }_{16}$ En un total de 4.500 municipios, la media encontrada fue de 1,99 habitantes por vivienda para 1.655 municipios del primer Brasil; de 2,15 habitantes por vivienda para 1.201 municipios del segundo Brasil, y la media de 2,35 habitantes por vivienda para 1.635 municipios del tercer Brasil. Si bien esta dimensión no ha sido incluida para los cálculos del Índice de Desarrollo Humano (IDH), la relación encontrada fue indiscutible: los índices empeoran a medida que transita del primer hacia el segundo y el tercer Brasil. 


\section{Estimación del grado de instrucción del electorado brasileño, considerando los electores de veinte años y más}

\begin{tabular}{|c|c|c|c|}
\hline & & Porcentaje & Porcentaje \\
\hline Sin instrucción y menos de un año de instrucción ............... & 14.257 .027 & 16 & \\
\hline De 1 a 3 años & 16.618 .725 & 18 & \\
\hline De 4 a 7 años & 28.503 .374 & 32 & 66 \\
\hline De 8 a 10 ańos & 11.826 .612 & 13 & \\
\hline 11 años y más & 19.241 .947 & 21 & \\
\hline TOTAL & 90.447 .685 & 100 & \\
\hline
\end{tabular}

FUENTE: IBGE, PNAD, 1996.

Las diferencias de grado de instrucción del electorado son también enormes cuando, más allá de las diferencias regionales, tenemos en cuenta el hecho de encontrarse en las capitales de estados o en los municipios del interior. Tales diferencias son más destacadas si consideramos las regiones clasificadas según los tres Brasiles.

Electores sin instrucción o con menos de tres años de instrucción en Brasil, viviendo en capitales o en municipios del interior

\begin{tabular}{|c|c|c|c|}
\hline & & $\begin{array}{c}\text { Porcentaje } \\
\text { del total }\end{array}$ & $\begin{array}{c}\text { Porcentaje } \\
\text { en el interior }\end{array}$ \\
\hline \multicolumn{4}{|l|}{ Primer Brasil } \\
\hline 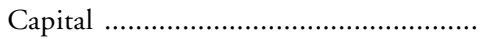 & 1.989 .110 & & \\
\hline 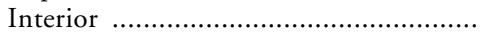 & 9.528 .740 & & 31 \\
\hline 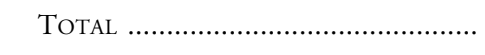 & 11.517 .850 & 37 & \\
\hline \multicolumn{4}{|l|}{ Segundo Brasil } \\
\hline 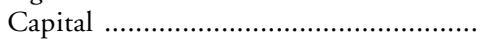 & 541.682 & & \\
\hline Interior & 4.841 .671 & & 16 \\
\hline TOTAL & 5.383 .353 & 17 & \\
\hline \multicolumn{4}{|l|}{ Tercer Brasil } \\
\hline 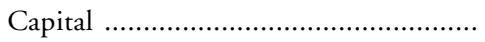 & 1.505 .972 & & \\
\hline Interior & 12.318 .203 & & 40 \\
\hline 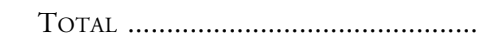 & 13.824 .175 & 54 & 87 (total/Brasil) \\
\hline
\end{tabular}

FUENTE: IBGE, PNAD, 1996. 
Del total de electores con muy baja instrucción - hasta tres años de escolaridad-, el 46 por 100 están en el tercer Brasil, estando el 40 por 100 de ellos en municipios del interior de esta sub-región. En números absolutos, esto significa un contingente aproximado de 14.000.000 de individuos con veinte años o más. El dato más impresionante es que del total de los 31.000.000 de electores de muy baja instrucción, el 87 por 100 de ellos se encuentran en el interior, y el 40 por 100 en municipios del interior de los estados del Norte y Nordeste del país, que constituyen la sub-región del tercer Brasil. La pregunta que nos hacemos, en el contexto de la presente discusión, es la siguiente: ¿Cuáles son las oportunidades de la población que vive en el gran interior del país para organizarse de modo autónomo en la defensa de sus intereses?

Un dato complementario al presentado más arriba, y que analizaremos enseguida, está relacionado con la distribución de los electores con una instrucción por encima de once años en las tres subregiones analizadas.

Estimación del electorado con veinte años o más, con instrucción correspondiente a once años y más en Brasil

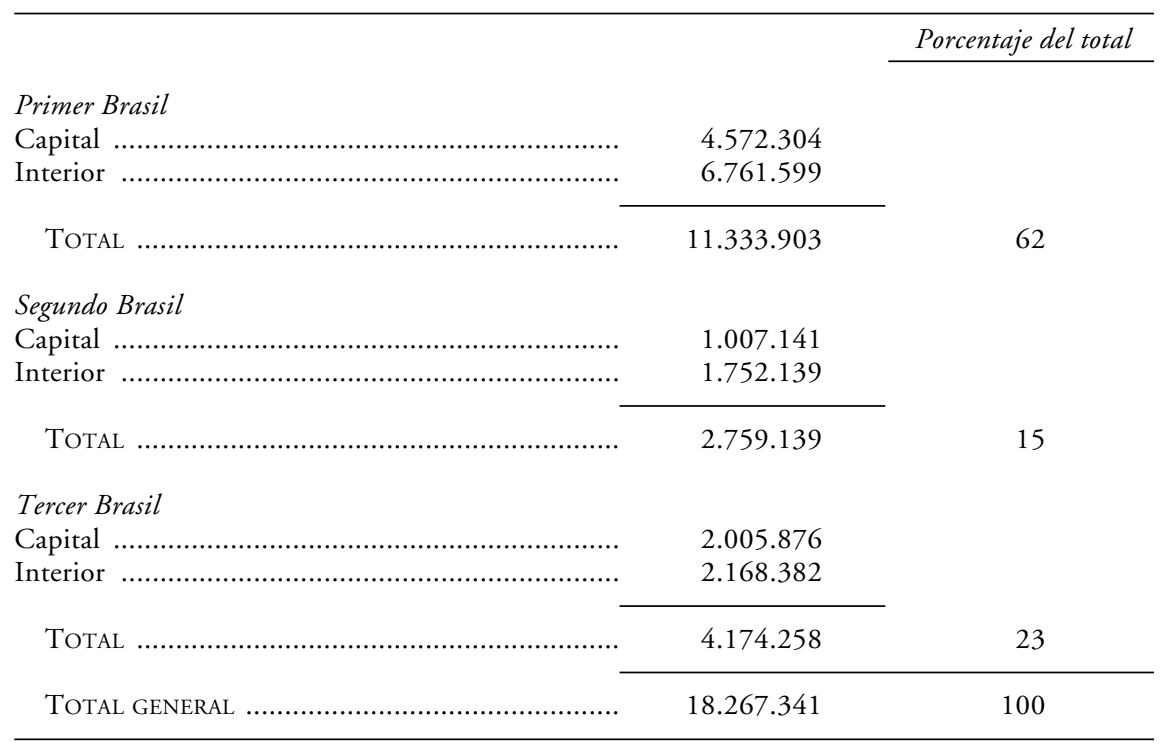

Fuente: IBGE. Tabulação Especial de la PNAD, 1996.

Es, pues, en el primer Brasil en el que se encuentra el 62 por 100 de los electores con escolaridad correspondiente a once años y más. No es por casualidad que esto se relacione fuertemente con el dato presentado anteriormente sobre organización política: ésta es mayor en los estados de la sub-región del 
primer Brasil, en las regiones de mayor desarrollo. No queremos afirmar con esto que el desarrollo lleva a la superación de las arcaicas estructuras del poder político. El desarrollo es apenas una de las dimensiones a ser considerada, tal vez la más evidente. Con todo, otros factores son decisivos: la forma de ocupación de las tierras en cada región, las formas tradicionales de organización política, los elementos de socialización política, una cultura marcada por mayor solidaridad y confianza en las relaciones en contraposición a las culturas consideradas.

\section{El electorado brasileño: una interpretación}

Como muestran los datos sobre el electorado brasileño, el gran cleavage se encuentra entre el elector de muy baja escolaridad y el elector de alta escolaridad. Tal cleavage responde a situaciones objetivas de vida debido a la fuerte concentración de renta del país, con enormes consecuencias para los resultados electorales. Por un lado, un electorado de mayor centralidad, con situaciones de vida y de escolaridad más privilegiadas; de otro, el electorado popular, el más numeroso, correspondiendo en términos numéricos al 60 por 100 del total.

El electorado de mayor centralidad se encuentra más expuesto a las informaciones, con mayores condiciones para la construcción de su universo cognitivo, lo que le posibilita integrar issues políticos y elaborar un diagnóstico sobre las cuestiones políticas del momento. En vez de ser mera masa de maniobra, retrocede y es capaz de evaluar críticamente la ideología dominante. Tiene preferencias, hace elecciones, pasa a reaccionar selectivamente y se orienta con objetivos de largo plazo. Los factores de cognición, junto a una identidad más estructurada, conllevan una mayor autonomía política. Está constituido por, aproximadamente, 30.000 .000 de individuos de un total de 100.000 .000 (Reis, 1996).

El grueso del electorado brasileño está muy lejos de ese modelo de autonomía, lucidez e información. Son aproximadamente 70.000.000 de electores que no pasan de los siete años de escolaridad. Se encuentran dispersos en los 5.500 municipios brasileños, la mayoría de ellos constituidos por localidades muy pequeñas. Hay en el país, por lo menos, 1.500 municipios con menos de 5.000 habitantes, más 1.400 donde encontramos de 5.000 a 10.000 habitantes. Es decir, el 65 por 100 de los municipios brasileños son localidades muy pequeñas en población y en electorado; pero, debido a su número, aproximadamente el 50 por 100 del electorado brasileño vive en municipios de menos de 50.000 habitantes y, en general, municipios de calidad muy mala. Ese numeroso electorado popular se encuentra también en las bolsas de pobreza de las grandes metrópolis, igual que en los municipios de las regiones rurales y semi-rurales, particularmente del tercer Brasil. En ellas, aunque haya elecciones regulares, la norma es la subordinación política a cambio de recursos materiales, siendo difícil la renovación de las élites. La subordinación política del elec- 
torado popular del interior del país se manifiesta, en los mayores centros urbanos, en volatilidad: no siempre es posible saber cuál de los «lados» en disputa le parecerá más conveniente para la resolución de sus problemas más inmediatos (Reis, 1976, 1989, 1991). El populismo urbano es la base de la movilización electoral en estos grandes centros.

\section{Renovación de las élites y cambio politico}

Sea por los canales numéricos —el sistema electoral y partidario—, sea por los canales corporativos, el acceso al poder sólo se abre a las nuevas élites progresivamente. Los criterios de reclutamiento son selectivos, además de presentar una relación directa entre la adquisición de poder formal y la participación informal en la red de relaciones de la élite dominante. Véase, por ejemplo, el reclutamiento para los cargos de las diferentes áreas ministeriales, de los más altos a los segundos y terceros escalones del edificio burocrático-representativo gubernamental, posiciones seniors que traen oportunidades para un discreto ejercicio del poder. En todo el conjunto de la burocracia pública estatal esos cargos más altos son ocupados por las élites burocráticas, que se constituyen a imagen del poder político estructurado en el centro político con la fuerza corporativa de los líderes de la periferia del sistema.

Otro ejemplo de los más evidentes se refiere a las instancias del Poder Judicial. Con una compleja estructura de poder, una rígida jerarquía en la provisión de sus incontables cargos a pesar de los concursos públicos, la ascensión a sus más altas instancias pasa por canales de preferencias de orden valorativa, social y política conforme al sistema de intereses de las élites en el poder. Los grupos corporativos del Poder Judicial presentan las mismas características de los grupos oligárquicos cerrados. Los procedimientos de nombramiento y promoción acaban siendo aquellos que reproducen el cuerpo directivo a su propia imagen, siendo la entrada en los grupos de la élite del Judicial dependiente de los intereses políticos y de la red de relaciones, en los mismos moldes de la ascensión en la carrera del Legislativo y el Ejecutivo.

Con la ascensión de algunos líderes de la sociedad organizada en ciertas esferas del Estado, se observa la constitución de lo que Reis llama «anillos de contrapeso", en oposición a la ascensión que se da por los "anillos burocráticos», convirtiendo la participación en la élite política por las vías corporativas en un mecanismo institucional nuevo para aquellos que, incluso sin gozar de atributos originales del capital social, consiguen ascender por la vía de la representación corporativa de intereses.

En este espectro, hay otros cambios en curso en el nivel organizacional y que tienen impacto sobre la renovación de las élites. No obstante el alarde por la evidente proliferación de las Organizaciones No Gubernamentales, algunas de ellas realmente eficaces, articulándose de modo dinámico, descentralizado y flexible entre los recursos del Estado y la sociedad, otras, con todo, se convir- 
tieron en órganos de poder o consejos alternativos, constituyendo, en palabras de Castells (1998), «fantasías políticas». Pero, sin duda, ellas son un ejemplo de la reconstitución de nuevas formas de instituciones políticas que intentan establecer la relación entre las identidades particulares y el mundo de flujos de poder, con el fortalecimiento de la sociedad. La expectativa es que esos nuevos actores puedan expresarse en el ámbito del Estado y constituir uno más de los elementos de ruptura con el poder secular oligárquico de estilo tradicional.

\section{Élites y politicas redistributivas}

El Gobierno de Fernando Henrique Cardoso (1994/1998 y reelegido en 1998) firmó numerosas políticas de desarrollo social para atender los segmentos poblacionales hasta ahora descuidados por las políticas gubernamentales. Una breve mención puede ayudarnos a entender cuáles son los mayores desafíos de cualquier Poder Ejecutivo brasileño en el sentido de implantar reformas que, al final, colaborarían en la erosión de la base de la dominación tradicional y de la política oligárquica. Lo primero a destacar es el Plan Real, un esfuerzo de reorganización de la economía que arrancó con el control inflacionario. La estabilidad de los precios fue el punto de partida, elaborándose a partir de ahí un amplio plan de reformas para la consolidación de la apertura económica buscándose activamente una reinserción del país en el comercio y en el sistema de relaciones internacionales. La orientación gubernamental básica es la de la desestatización: cambiar el papel del Estado empresario por el de Estado regulador. Otra medida fue la apertura al capital privado en los sectores de la infraestructura: puertos, telefonía, entre otros. El sistema financiero sufrió una reestructuración, cayendo del 12 al 7 por 100 la participación del sector financiero en el PIB, con bancos extranjeros entrando en el sistema. El ajuste fiscal, particularmente el ajuste de la Seguridad Social, se volvió central, siendo, históricamente, uno de los sectores que actúa como mecanismo de distribución regresiva de la renta, por el hecho de contener dos orientaciones: una, para funcionarios públicos, y otra, para trabajadores de la iniciativa privada, siendo el valor medio de las jubilaciones del sector privado 1,8 veces el salario mínimo (12.000.000 de personas), y el valor medio de las jubilaciones del sector público, 14 veces el salario mínimo.

La reestructuración productiva llevó a la reducción del número de empleados en la industria, ahora compensada con la expansión del sector de servicios, los de altísima cualificación en centros desarrollados y en el turismo tradicional y en el ecoturismo. Se cuidó de abrir nuevas fuentes de trabajo como la agricultura familiar, siendo los asentamientos rurales el soporte de la política agraria. Para afrontar la cuestión de la deuda educacional, se implementó la política que tiene como base corregir las distorsiones de la enseñanza fundamental, reinsertando la población en edad escolar y recuperando los salarios de los profesores que venían declinando desde la década de 1970, con pérdidas 
mensuales acumuladas que alcanzaban, en 1996, el 15 por 100 de los niveles anteriores.

El sistema público de salud era otro desafío, con necesidades que iban desde la reducción de la tasa de mortalidad infantil en las regiones más carentes, hasta el combate contra el hambre, la desnutrición infantil y la recuperación de los hospitales públicos.

Las políticas de desarrollo y las reformas pretendidas, encuentran obstáculos de naturaleza política para su implementación. Si los bienes públicos siempre fueron materia prima de operaciones clientelistas, los políticos que dependen de la protección (patronagem) serán opositores de algunas de las reformas propuestas. El dilema político no es nuevo, hasta el punto que los presidentes asumieron, desde 1930, que la realidad a ser enfrentada es la de «incentivos cruzados»: si el desarrollo implica cambios en la distribución de recursos que encontrarán resistencias del status quo, habrá tensiones entre objetivos programáticos y realidad política (Geddes, 1996). En este sentido el desarrollo sería operacionalizado para algunas áreas, aislándose parte de la burocracia del Estado. Los conflictos entre burócratas y políticos son evidentes.

Getúlio Vargas fue el autor de la primera innovación estratégica, cuando centralizó el Estado, expandió y reformó su burocracia. Inventó superministerios vinculados directamente al presidente, impuso exámenes para el reclutamiento del personal administrativo, creó nuevas categorías de funcionarios más habilitados, aisló algunas agencias de los conflictos de intereses. Ganó enemigos, tanto en el plano federal como local, al implantar un Estado desarrollista reconstruyendo la burocracia y su élites por criterios de competencia. Para todo ello, tuvo que negociar con los partidos y políticos clientelistas, con el Congreso, consciente de que, sin esas negociaciones, las medidas desarrollistas no encontrarían apoyo político. Jânio Quadros (1960) fracasó en la conquista del Congreso, y João Goulart (1963) sucumbió ante las demandas trabajadoras y de patronagem. Queda claro que el Estado se dividía entre una burocracia tradicional y bolsas "de eficiencia» — un segmento aislado que asumiría las reformas y las políticas de desarrollo_- Kubitschek (1955-1960), por ejemplo, reorganizó fondos financieros para objetivos específicos a partir de impuestos sobre petróleo y electricidad, fondos que eran depositados en el Banco Nacional de Desarrollo Económico y no incluidos en el presupuesto federal, evitando su utilización por el Congreso. Las agencias públicas que lograron autonomía del juego político tradicional desempeñaron un importante papel en la implementación del Programa de Metas de Kubitschek. Aisló y protegió recursos humanos y materiales, administrando el resto del sistema político de modo tradicional. Con una cara populista y otra desarrollista, fue un estratega victorioso de las reformas.

Es en este cuadro donde el actual Gobierno de Fernando Henrique Cardoso también diseña sus estrategias para las reformas anteriormente propuestas. La burocracia desempeña un papel crucial en la implementación de programas que promoverán cambios económicos y sociales. Pero esto no es todo. Los pac- 
tos institucionales, como los que rigen la legislación proporcional de lista abierta, son fuentes de clientelismo y corrupción. Y el presidente se enfrenta a obstáculos políticos que se oponen a los cambios. Esto a pesar del relativo consenso en torno a que la reducción de los conflictos originados de las desigualdades regionales y sociales, además de enfrentar los problemas apuntados, precisa aprovechar otras vías de cambio, deseadas y reconocidas como medio posible para alcanzar una mayor eficiencia. Pueden resumirse en cuatro puntos: a) El incentivo a la participación política con el reconocimiento de las colectividades «desiguales»; b) cambios en la legislación de la representación política; c) el incentivo a los mecanismos de descentralización, con la participación efectiva de las colectividades; d) la reafirmación en algunas tareas y objetivos del Estado. El consenso también se cierra en cuanto a la certeza de que, si las élites tradicionales todavía no han practicado políticas redistributivas, los cambios políticos son cruciales. Como nos decía Hobsbawm (1994), en conferencia reciente, con sabias palabras:

«El problema básico del globo en las próximas décadas no será el crecimiento de la riqueza y sí la distribución equitativa de la riqueza producida. La redistribución requiere, esencialmente, que algunos deben perder y otros deben ganar. Las inversiones infraestructurales requieren más recursos para la educación, por ejemplo, para la protección ambiental, que también requiere interferencia en el libre mercado. Y, para hacer las cosas esenciales, tenemos que tener partidos y élites comprometidas con la redistribución. Y no apenas por razones de equidad: tenemos que tener una infraestructura de desarrollo y de redistribución social de la renta por fuertes razones económicas, para el equilibrio del sistema de competición capitalista.»

Se espera que, en el movimiento de la dialéctica de la historia, las élites tradicionales tengan que convivir con otra élite de origen distinta. Si la política democrática es un constraint sobre los recursos y son las élites en disputa que deciden hacia dónde y cómo serán aplicados, esperamos que, en la disputa electoral y partidaria, ocurran cambios suficientes para una transformación política que posibilite otra configuración de las élites, comprometidas con la redistribución de la renta y con la democracia social. 


\section{BIBLIOGRAFÍA}

Avelar, Lúcia: "Clientelismo de Estado e política educacional brasileira», Revista Educação e Sociedade, n. ${ }^{54}$, CEDES/Papirus.

Avelar, Lúcia, y Lima, Fernão (2000): «Lentas mudanças: voto e política tradicional», Revista Lua Nova, São Paulo, CEDEC, junio.

Azevedo, S., y Prates, Antonio Augusto (1996): «Planejamento participativo, movimientos sociais e ação coletiva». Trabajo presentado en el Seminario Temático «Planejamento, pobreza urbana e representação política».

Bezerra, Marcos (1999): Em nome das "bases»-Política, favor e dependência pessoal, Río de Janeiro, Relume-Dumará.

CANEDO, Letícia (1993): Caminhos da memória: parentesco e poder, Mimeo.

Carvalho, José Murilo: «Estudos de poder local no Brasil», Revista Brasileira de Estudos Politi$\cos$, n. ${ }^{\circ} 25$, Belo Horizonte.

Cintra, Antônio Octávio (1974): «A política tradicional brasileira: uma interpretação das relaçōes entre o centro e a periferia», Cadernos de Ciência Política, Belo Horizonte.

Della PorTa, Donatela (1996): Lo scambio oculto-casi de corruzione política in Itália, Societá Editrice Il Mulino.

Eisenstadt, S. N., y Lemarchand (1981): Political clientelism, patronage and development, Londres, Sage Publications.

Fox, Jonathan (1994): «The difficult transition from clientelism to citizenship. Lesson from Mexico", World Politics.

Geddes, Barbara (1996): Politican's dilemma-Building State capacity in Latin America, Berkeley, Los Ángeles, Londres, University of California Press.

Hagopian, Frances (1996): Traditional politics and regime change in Brazil, New York, Cambridge University Press.

KAUFMAN, Robert (1974): «The patron-client concept and macro-politics: prospects and problems", Comparativity Studies in Society and History, vol. 16:3.

KeCK, Margareth (1991): «PT: a lógica da diferença», São Paulo, Ática.

LINZ, Juan, y STEPAN, Alfred (1996): Problems of democratic transition and consolidation, Baltimore and London, The John Hopkins University Press.

Leff, Nathaniel (1977): Politica econômica e desenvolvimento no Brasil, São Paulo, Perspectiva.

MARTins, José de Souza (1994): "Clientelismo e corrupção no Brasil contemporâneo», en O poder do atraso, São Paulo, Hucitec.

MAINWARING, S., y VIOLA, E. (1987): «Novos movimientos sociais: cultura, política e democracia: Brasil e Argentina», en Seherer-Warren y P. Krische (orgs.), Uma revolução no cotidiano?, São Paulo, Brasiliense.

Pizzorno, Alessandro (1975): «Introducción al estudio de la participación política», en A. Pizzorno, Kaplan y M. Castells, Participación y cambio en la problemática contemporánea, Ediciones Siap-Planteos.

Roniger, L., y Gunes-Ayata, A. (1994): Democracy, clientelism and civil society, Colorado, Lynne Rienner Publischers.

SCHMIDT, S. W., et alii (ed.) (1977): Friends, followers and factions, Berkeley, University of California Press.

REIS, Fábio Wanderley (1974): «Solidariedade, interesses e desenvolvimento político», Cadernos de Ciência Política, marzo.

- (1989): «Partidos, ideología e consolidação democrática», en F. W. Reis, O’Donnell, A democracia no Brasil: dilemas e perspectivas, São Paulo, Vértice.

- (1996): «Cidadania, Estado e mercado: democracia social e democracia política no processo de transformação capitalista». Trabajo presentado en el coloquio Democracia política y democracia social, México, Centro de Estudios Sociológicos.

RELATÓRIO SOBRE O DESENVOLVIMENTO HUMANO NO BRASIL (1998): PNUD/IPEA. 
STEPAN, Alfred (1999): «Towards a new comparativity analysis of democracy and federalism», Mimeo, Oxford.

Vianna, Oliveira (1999): Instituições políticas brasileiras, Biblioteca Básica Brasileira, Senado Federal.

(Traducido del portugués por Alberto Begué AguAdo)

\begin{abstract}
This article seeks answers in relation to the refusal of the Brazilian political elites to introduce socio-redistributive policies, and thereby calls into question the reasons for the increase in social inequalities in a rich country such as Brazil. The analysis of these elites highlights the existence of patronage style practices carried out by the State itself and in which the regional distribution of elites has taken on special relevance since the $19^{\text {th }}$ century. Over time, the increasingly complex nature of Brazilian society has opened up the way for a certain degree of political change which has brought with in the appearance of political organizations and associations, mainly in the richest states such as São Paulo and Rio de Janeiro. Nevertheless, redistributive imbalances are still maintained between the three Brazils. The second part of the study places these earlier elements in relation to the educational level of the Brazilian electorate, in an analysis of the possibilities existing for a renewal of élites and deep political change, as it seems we are to witness the effective application of redistributive policies which may make it possible to improve the living conditions of the majority of the Brazilian population.
\end{abstract}


NOTAS DE INVESTIGACIÓN 